\title{
ESTIMACIÓN DE PARÁMETROS GENÉTICOS PARA PESO Y TALLA A DIFERENTES EDADES EN YAMÚ (Brycon amazonicus)
}

\section{ESTIMATION OF GENETIC PARAMETERS FOR BODY WEIGHTS AND LENGTH TO DIFFERENT AGES IN YAMU (Brycon amazonicus)}

\begin{abstract}
Felipe Bernal $^{1}$, Fernando Gallego ${ }^{2}$
${ }^{1}$ Zootecnista. cMSc, Grupo de Investigación sobre Reproducción y Toxicología de Organismos Acuáticos -GRITOX-, Instituto de Acuicultura -IALL-, Facultad de Ciencias Agropecuarias y Recursos Naturales. Universidad de los Llanos, Villavicencio, Meta, Colombia, e-mail: gebernal@udca.edu.co; ${ }^{2}$ Zootecnista, Ph.D., Profesor Titular, Facultad de Ciencias Pecuarias, Programa de Zootecnia. Universidad de Ciencias Aplicadas y Ambientales U.D.C.A, Bogotá, Colombia, e-mail: fgallego@udca. edu.co
\end{abstract}

Rev. U.D.C.A Act. \& Div. Cient. 19(1): 123-130, Enero-Junio, 2016

\section{RESUMEN}

Dado que la finalidad de los programas de mejora genética es manipular la frecuencia de los genes que intervienen en la expresión de una característica, es importante comprender los cambios asociados que ocurren en otros caracteres, debido a la selección y en qué medida se transmiten a la descendencia. El presente estudio reporta, por primera vez en yamú (Brycon amazonicus), la estimación de parámetros genéticos, para las variables peso y talla, a los 25 , 40 y 55 días post-eclosión. Un total de 900 progenies de la reproducción de seis parejas, fueron analizadas por medio del paquete estadístico SAS 9,3 (SAS Institute). Se asumió un modelo lineal general, con inclusión de los efectos fijos, como factores de entorno y los efectos aleatorios, como factores genéticos. Para estimar los componentes de varianza y covarianza, se utilizó el procedimiento VARCOMP, bajo el método de máxima verosimilitud restringida (REML). La heredabilidad promedio estimada para peso fue 0,37 $\pm 0,22$ y $0,58 \pm 0,30$, para talla. La correlación genética y fenotípica entre peso y talla fue positiva, en promedio $0,39 \pm 0,19$ y $0,55 \pm 0,14$, respectivamente. La correlación para peso y talla fue positiva e incremental en función de la edad. Estos resultados sugieren que la selección directamente por talla sobre los 55 días post-eclosión, incrementará también el peso indirectamente. Este estudio destaca el potencial genético para mejorar la industria del yamú, una vez se avance en las áreas de morfo-fisiología, nutrición, reproducción y sanidad de la especie.

Palabras clave: Reofílico, heredabilidad, correlación genética y fenotípica, máxima verosimilitud restringida.

\section{SUMMARY}

Since the purpose of breeding programs is to manipulate the frequency of genes involved in the expression of a characteristic it is important to understand the associated changes that occur in other characters due to the selection and to what extent they are transmitted to the progeny. The present study reports for the first time in yamú (Brycon amazonicus), an estimation of genetic parameters for the variables weight and height at 25,40 and 55 days post-hatch. A total of 900 progenies of six couples were analyzed using the statistical package SAS 9.3 (SAS Institute). A general linear model including the fixed effects of environmental factors and genetic factors as random effects were assumed. To estimate the components of variance and covariance the VARCOMP procedure was used under the restricted maximum likelihood method (REML). The average weight estimated heritability was $0.37 \pm 0.22$ and $0.58 \pm 0.30$ for size. Genetic and phenotypic correlation between weight and height was positive on average $0.39 \pm 0.19$ and 0.55 \pm 0.14 , respectively. The correlation for height and weight was positive and increasing, depending on age. These results suggest that direct selection by size at around 55 days posthatch, will also increase the weight indirectly. This study highlights the genetic potential to improve yamú industry, once progress is made in the areas of morpho-physiology, nutrition, reproduction and health of the species.

Key words: Rheophilous, heritability, genetic and phenotypic correlation, restricted maximum likelihood. 


\section{INTRODUCCIÓN}

El yamú (Brycon amazonicus) es un pez reofílico, nativo de Brasil, de Colombia, de Guyana y de Perú, muy extendido en el oeste de la cuenca del río Amazonas, el río Orinoco y el río Essequibo de Guyana. Es de excelente calidad de carne, rápido crecimiento y demanda en aumento, de acuerdo con Arias (2006).

Esta especie ha brindado, desde sus inicios, una excelente alternativa para los productores; sin embargo, el yamú no ha logrado posicionarse comercialmente, puesto que aun representa menos del $4 \%$ de la actividad piscícola, de acuerdo con los reportes de Merino et al. (2013), debido a múltiples factores productivos como: la larvicultura y el alevinaje, donde el canibalismo es muy acentuado, según de Souza et al. (2014); la nutrición, donde se requiere de investigaciones que acerquen a sus requerimientos, como afirma Urbinati et al. (2014); los reproductivos, de igual forma, requieren atención, para romper la obtención de semilla bianual, lo que ha generado una producción inconstante durante todo el año, de acuerdo con Zanuzzo et al. (2015); asimismo, Arias (2006) resalta la importancia de las investigaciones en el área de la genética aplicada, como uno de los factores más urgentes y esenciales, no solo para el desarrollo y mejoramiento genético de la especie, sino para el de la piscicultura continental, buscando, de esta manera, conformar e impulsar los primeros eslabones necesarios para el fortalecimiento de la cadena productiva, que conforma una gran parte de la población de piscicultores de la familia Bryconidae, tratando de involucrar a muchos expertos en el tema del progreso de esta especie y, de este modo, generar empleo y desarrollo socioeconómico, no solo en los piscicultores, sino en la sociedad en general.

En este sentido, encaminar este sector, con base en el conocimiento genético de las especies nativas, como el yamú, Gjedrem (2012) explica que, dado que la genética de cualquier carácter métrico gira en torno al estudio de su variación y que son éstas las que permiten que la población cambie genéticamente, en la dirección deseada, como resultado del proceso de selección; por lo tanto, Gallego (2001) reitera la necesidad de conocer en qué medida la varianza fenotípica es explicada por la varianza debida a los genes, su evolución con la edad de los animales y la correlación genética en aquellas especies y características de interés.

De acuerdo a lo anterior, este estudio tuvo como objetivo estimar la heredabilidad, la correlación genética y fenotípica, para las variables peso y talla, a edades tempranas de desarrollo.

\section{MATERIALES Y MÉTODOS}

La fase experimental, se llevó a cabo en la empresa Langostinos del Llano Ltda., ubicada en la vereda Caney Medio, en el municipio de Restrepo, departamento del Meta, cuyas coordenadas son $4^{\circ} 16^{\prime} 8^{\prime \prime}$ de latitud Norte y $73^{\circ} 32^{\prime}$ '55" de latitud Este, a 18 kilómetros de la vía que conduce de la ciudad de Villavicencio, al municipio de Cumaral. La finca cuenta con una extensión de 9 hectáreas, se encuentra a 450 metros sobre el nivel del mar, temperatura media anual de $26^{\circ} \mathrm{C}$, que puede variar de 17 a $34,4^{\circ} \mathrm{C}$, una humedad relativa promedio de $83 \%$, que puede llegar hasta $93 \%$ en época de lluvia y $56 \%$ en época seca y una precipitación anual promedio de $5062 \mathrm{~mm}$.

Para la obtención del material experimental, se utilizó el modelo de parejas de apareamientos simples, descrito por Campos (2004), que se refiere a su uso en animales multíparos. El modelo de 6 familias de hermanos completos fue conformado por seis parejas de 6 machos y 6 hembras de reproductores independientes y excluyentes, provenientes de la misma empresa, nacidos y criados bajo condiciones de cautiverio.

Los parentales, se reprodujeron por inducción hormonal, bajo los criterios descritos por Daza et al. (2005). Debido a que no se utilizó ningún tipo de identificación, una vez obtenidas las familias, fueron mantenidas separadamente en estanques en tierra, hasta alcanzar la talla de $2,5 \mathrm{~cm}$, a una edad de $13 \pm 1,5$ días; luego, fueron capturados y dispuestos separadamente en piletas rectangulares, a una densidad no mayor a 0,25 animales por litro, durante cuatro días; posteriormente, se seleccionaron 150 individuos de talla homogénea, para cada una de las 6 familias; cada una de estas fue dividida en dos tanques de manera aleatoria, para un total de 12 tanques, 2 por familia y 900 animales utilizados. Les fue brindado un periodo de adaptación de $7 \pm 1,5$ días en las condiciones experimentales, para así darles la posibilidad a los peces para expresar su tasa de crecimiento, al inicio del primer pesaje.

El periodo experimental, se desarrolló en un recinto aislado, bajo condiciones de laboratorio, provisto de un aireador, que permitió el control y la estabilidad de la concentración de oxígeno disuelto, temperatura y calidad de agua y tanques de polipropileno, con capacidad de 500L. Inicialmente, fueron limpiados y desinfectados, dentro de las instalaciones, todos los elementos que tendrían contacto con los animales, como tanques, tapas, tuberías, llaves y piedras difusoras.

Los pesajes, se realizaron en una balanza electrónica analítica de precisión, desde 0,0001g, de marca SARTORIUS BASICPLUS, modelo BP 221C y para las mediciones de talla, se utilizó un ictiómetro, con indicadores cada $1 \mathrm{~mm}$. 
La alimentación, se basó en cuatro fases, como se muestra en la tabla 1. La transición de una a otra, se realizó cuando al menos una de las unidades experimentales, de cualquier grupo familiar, lograra entrar en el siguiente rango de peso. El alimento tuvo $45 \%$ de proteína bruta y la frecuencia alimenticia fue de cuatro veces al día. En las dos primeras fases, el alimento fue pulverizado, después se ofreció peletizado y de un tamaño fácilmente consumible.
En cuanto a la calidad del agua, a diario se registró su temperatura, utilizando un termómetro digital MARTINI@ instruments EC59. Semanalmente, se efectuaron determinaciones de oxígeno disuelto, $\mathrm{pH}$, amonio, dureza y alcalinidad, mediante Kit comercial Kit Hach FF1-A® - Hach Company.

Los pesajes y las mediciones de longitud total de los individuos, se verificaron cada 15 días, a partir de la primera

Tabla 1. Alimentación por fases de crecimiento y consumo promedio durante el estudio.

\begin{tabular}{|c|c|c|c|}
\hline Peso promedio $\mathbf{( g )}$ & Edad/días & Gramos/Pez /Día & Consumo $\mathbf{( g )}$ \\
\hline $0,356-0,95$ & 25 & 0,0783 & 0,626 \\
\hline $1,0-2,584$ & 41 & 0,2153 & 1,722 \\
\hline $2,65-4,00$ & 49 & 0,3657 & 2,925 \\
\hline $4,10-6,00$ & 57 & 0,505 & 4,04 \\
\hline
\end{tabular}

(Vásquez, 2004)

toma de datos de la muestra piloto; para la realización de estos procedimientos, los peces fueron atrapados en redes y anestesiados, usando una solución de benzocaína a 50ppm, reportada por Velasco-Santamaría et al. (2008). El número de peces muestreados por cada familia fue de 14, sumado por los dos tanques dispuestos por familia; este tamaño de muestra fue establecido al inicio del estudio, siguiendo la metodología propuesta por McDonald (2008), utilizando la expresión $n=1 /((E /(t * C V)) 2+(1 / N))$, donde $n$, es el tamaño de la muestra; $E$, el porcentaje de error que se quiere asumir; $\mathrm{t}$, es el valor tabulado de $\mathrm{t}$ con $\mathrm{n}-1$ grados de libertad $(\mathrm{n}=$ número de mediciones en una muestra piloto) y un nivel de significancia $(\alpha)$; CV, es el coeficiente de variación observado en la muestra piloto y $\mathrm{N}$, el tamaño de la población; para ello, se asumió un $5 \%$ de error y un coeficiente de variación de $22 \%$, el cual, fue determinado mediante la muestra piloto de 40 individuos de cada grupo de apareamiento, es decir, 20 de cada tanque. Esta muestra piloto no se utilizó posteriormente en el estudio, debido a que está demostrado, en anteriores investigaciones reportadas por Maluwa \& Gjerde (2006) y Vine et al. (2004), que los animales sufren un elevado estrés, incrementando la varianza del error en el modelo. Diariamente fue registrado el número de animales muertos en cada tanque y ajustado el nivel de la columna de agua, para garantizar la misma densidad de animales por volumen de agua, en todos los tratamientos.

Para el análisis de los datos obtenidos durante el desarrollo del estudio, se utilizó el paquete estadístico SAS 9,3 (SAS Institute). Las diferencias se consideraron significativas, si $P<0,05$ y no significativas, cuando $P>0,05$. La homogeneidad de la varianza en la prueba piloto fue comprobada mediante el estadístico Levene's F-test, con el procedimiento ANAVA. Las variables fueron sujetas al análisis de varianza usando el procedimiento GLM. La normalidad fue verificada por la prueba de Kolmogorov-Smirnov, con el procedimiento UNIVARIATE, que se usó también para calcular los estadísticos descriptivos de las variables. Para estimar los componentes de varianza y de covarianza, se utilizó el procedimiento VARCOMP, para modelos mixtos PROC MIXED, con el método de máxima verosimilitud restringida (REML). El modelo usado fue similar al empleado por García-Celdrán et al. (2015), donde se asumió un modelo lineal general con inclusión de los efectos fijos ambientales, como edad, época, alimentación, condiciones de laboratorio y el efecto genético como aleatorio, así: $\mathrm{Y}=\mathrm{X} \beta+\mathrm{Z} \mu+\mathrm{e}$, donde $\mathrm{Y}$, fue el dato registrado para la característica de estudio; $\beta$, el efecto fijo: ambiente controlado y $\mu$, el efecto aleatorio: factores genéticos, debidos al agrupamiento de hermanos completos. Las magnitudes de la estimación de la heredabilidad fueron interpretadas siguiendo la clasificación de Cardellino \& Rovira (1987), como: baja 0,05 $-0,19$; media $0,20-0,44$; alta $0,45-0,64$ y muy alta $>0.65$. La correlación fue clasificada como: baja 0,0 - 0,44; media $0,45-0,59$ y alta $0,60-1,0$. Las heredabilidad y correlaciones fueron estimadas siguiendo la metodología de apareamientos simples, propuesta por Becker (1975).

\section{RESULTADOS Y DISCUSIÓN}

Para los parámetros de calidad de agua, la temperatura promedio fue de $25,7 \pm 0,8^{\circ} \mathrm{C}$ durante el día, con un nivel de confianza del $95 \%$; los demás parámetros evaluados estuvieron constantes durante todo el estudio. El pH estuvo en 7,0 , la alcalinidad en $34,2 \mathrm{mg} / \mathrm{L}$, la dureza en $17,1 \mathrm{mg} / \mathrm{L}$ y la concentración de oxígeno disuelto en $90 \mathrm{~g} / \mathrm{L}$.

Los datos obtenidos muestran que el estudio se mantuvo dentro de las condiciones adecuadas para cultivo de peces 
dulceacuícolas, de acuerdo con Zaniboni-Filho et al. (2006) y según lo reportado por Sipaúba-Tavares et al. (2008) en un estudio con Brycon orbignyanus, demostrando un rendimiento apropiado en ambientes de cultivo, con características de calidad de agua, que comprenden temperaturas entre $\operatorname{los} 23$ y $30^{\circ} \mathrm{C}$, con mejores rendimientos entre 25 y $28^{\circ} \mathrm{C}, \mathrm{pH}$ entre 6,0 y 7,5, concentración de oxígeno disuelto superior a $4,5 \mathrm{mg} / \mathrm{L}$ y dureza $<200 \mathrm{CaCO}_{3} \mathrm{mg} / \mathrm{L}$.

Por otra parte, las condiciones de laboratorio permitieron un mejor control de las características del agua y del estado sanitario de los animales; se realizaron recambios con una frecuencia de dos veces por semana de máximo 30\% de la columna del agua, durante toda la etapa experimental, para que no se vieran afectados las condiciones del agua y el rendimiento de los animales.

Para las características de crecimiento, como peso y talla, la tabla 2 describe las variables de crecimiento, a 25, 40 y 55 días. Se comprobó la normalidad de los datos utilizando la prueba de Kolmogorov-Smirnov ( $\mathrm{P}>005)$, a excepción para los 25 días, que se rechazó este modelo probabilístico $(P<0.01)$. La homogeneidad fue aceptada para peso en todas las edades $(P>0.05)$ y rechazada para talla $(P<0.01)$.

Debido a la robustez del ANAVA frente al supuesto de normalidad, no se realizó transformación de la información, pese a obtener un $\mathrm{P}<0.01$, a los 25 días. El coeficiente de variación indicó que animales más jóvenes tienen mayor variabilidad en el peso que en la talla.

Así como se esperaba, el análisis de varianza explicó, de mejor manera, el modelo a los 40 y 55 días $\left(R^{2}>0.3\right)$. La prueba de homoscedasticidad ayudó a entender la variación de estas características, mostrando diferencia en las variables, indicando, desde el principio, que para la variable peso, existe una menor varianza entre las familias, lo que significa una menor magnitud de heredabilidad en la estimación respecto de la variable talla, la cual, mostró una mayor variabilidad y heteroscedasticidad de sus varianzas entre las familias (Caballero et al. 2003).

La estimación de heredabilidad, correlación genética y fenotípica para peso y talla, es presentada en la tabla 3. El rango de la estimación para heredabilidad fue de 0,04 a 0,58, para peso y de 0,30 a 0,77 , para talla, valores similares a lo reportado por Charo-Karisa et al. (2007), en tilapia nilótica, para talla entre 0,40 - 0,60 y también valores más altos para talla que para peso; en general, estas estimas estuvieron en una magnitud similar al rango reportado para otras especies acuícolas, que van de 0,10 a 0,34, aplicando REML, bajo la metodología de modelos mixtos para peso a diferentes edades (Gjedrem, 2012).

A pesar de que la heredabilidad -en este estudio- estuvo en rangos similares a los reportados para otras especies,

Tabla 2. Peso corporal y talla presentados como media \pm desviación estándar, a diferentes edades en yamú (Brycon amazonicus).

\begin{tabular}{|c|c|c|c|c|}
\hline \multirow{2}{*}{$\begin{array}{l}\text { Días } \\
\text { post- eclosión }\end{array}$} & \multicolumn{2}{|c|}{ Peso (g) } & \multicolumn{2}{c|}{ Talla (cm) } \\
\cline { 2 - 5 } & $\mu$ & CV & $\mu$ & CV \\
\hline 25 & $0,52 \pm 0,11$ & 21,97 & $3,38 \pm 0,25$ & 7,13 \\
\hline 40 & $1,50 \pm 0,38$ & 21,94 & $5,00 \pm 0,49$ & 8,20 \\
\hline 55 & $5,26 \pm 0,75$ & 12,71 & $7,60 \pm 0,49$ & 5,27 \\
\hline
\end{tabular}

Coeficiente de variación (CV)

Tabla 3. Heredabilidades resaltadas en la diagonal (Media \pm Desviación estándar), correlación genética (bajo la diagonal) y correlación fenotípica (sobre la diagonal), entre peso corporal y talla (longitud total), en yamú a diferentes edades.

\begin{tabular}{|c|c|c|c|c|c|c|}
\hline & Peso 25 & Talla25 & Peso40 & Talla40 & Peso55 & Talla55 \\
\hline Peso25 & $\mathbf{0 , 0 4} \pm \mathbf{0 , 0 6}$ & $0,44 \pm 0,16$ & $0,45 \pm 0,25$ & $0,49 \pm 0,11$ & $0,39 \pm 0,19$ & $0,52 \pm 0,18$ \\
\hline Talla25 & $0,07 \pm 0,13$ & $\mathbf{0 , 3 0 \pm 0 , 2 2}$ & $0,53 \pm 0,21$ & $0,51 \pm 0,15$ & $0,54 \pm 0,22$ & $0,52 \pm 0,26$ \\
\hline Peso40 & $0,29 \pm 0,25$ & $0,18 \pm 0,29$ & $\mathbf{0 , 5 8} \pm \mathbf{0 , 3 1}$ & $0,69 \pm 0,17$ & $0,45 \pm 0,25$ & $0,63 \pm 0,20$ \\
\hline Talla40 & $0,25 \pm 0,19$ & $0,41 \pm 0,15$ & $0,44 \pm 0,06$ & $\mathbf{0 , 6 8} \pm \mathbf{0 , 3 3}$ & $0,69 \pm 0,23$ & $0,95 \pm 0,27$ \\
\hline Peso55 & $0,32 \pm 0,24$ & $0,48 \pm 0,11$ & $0,33 \pm 0,21$ & $0,56 \pm 0,14$ & $\mathbf{0 , 5 0} \pm \mathbf{0 , 2 9}$ & $0,75 \pm 0,18$ \\
\hline Talla55 & $0,33 \pm 0,15$ & $0,43 \pm 0,09$ & $0,26 \pm 0,25$ & $0,82 \pm 0,26$ & $0,68 \pm 0,16$ & $\mathbf{0 , 7 7} \pm \mathbf{0 , 3 4}$ \\
\hline
\end{tabular}


se identificó un ligero aumento en las magnitudes a mayor edad, debido, posiblemente, a que los componentes genéticos están en función de las frecuencias genéticas y pueden diferir de una población a otra. Según Nguyen et al. (2012) es de esperar que poblaciones de censo reducido, que han perdurado durante un tiempo, lo suficientemente largo como para alcanzar un grado considerable de fijación, muestren heredabilidades menores, que las de censo elevado, como algunas especies nativas.

Las correlaciones genéticas y fenotípicas entre peso y talla fueron muy variables, de 0,07 a 0,68 y de 0,44 a 0,75, respectivamente; estas fueron incrementando su magnitud respecto aumentaba la edad, debido a factores ambientales, efectos familiares que fueron disminuyendo progresivamente con la edad y, a su vez, posiblemente, a que la especie no ha sufrido manipulación de selección para estos caracteres, lo cual, puede significar que existe a edades tempranas un grupo mayor de genes que intervienen en sus expresiones, disminuyendo su herencia correlacionada (Campos, 2004). Debido a que la magnitud de las correlaciones entre características de crecimiento son muy importantes en el orden de los parámetros genéticos para tomar decisiones en los procesos de selección genética, los resultados obtenidos, en este estudio, sugieren que la pretensión de seleccionar a edades avanzadas para aumento de longitud, con el propósito de elevar indirectamente el peso, es una alternativa justificable, máxime en condiciones de campo, cuando no está disponible una balanza de precisión y tiene un bajo coeficiente de variación; por consiguiente, es más reiterado la variable peso, lo que indica mayores posibilidades en la intensidad de selección. Una observación similar fue hecha por Navarro et al. (2009a).

De igual manera, las correlaciones entre peso y talla son estimaciones constantes entre especies, mostrando valores generalmente altos y positivos, como los encontrados por Haffray et al. (2014) y Whatmore et al. (2013), en un estudio con cola amarilla (Seriola lalandi), que reporta para peso y longitud una correlación genética de 0,57 $\pm 0,28$. Asimismo, Navarro et al. (2009b), en dorada (Sparus auratus), reporta las correlaciones estimadas entre las variables peso y talla a diferentes edades, de 0,96 a 0,99, para diferentes edades evaluadas; sin embargo, en el presente estudio, estas no fueron significativamente importantes a la edad de 25 días, a excepción de la correlación entre las dos variables a los 40 y 55 días, que fueron cercanas a 1 y que, posiblemente continúen hasta el tiempo de cosecha.

Por otra parte, en cuanto a las correlaciones genéticas y fenotípicas entre edades para peso fueron de 0,29 a 0,33 y de 0,39 a 0,75, respectivamente; para talla, de 0,41 a 0,82 y de 0,51 a 0,95 , respectivamente, datos que, según Vandeputte et al. (2008), representan importancia, desde que permita tomar una decisión sobre la preselección a edades tempranas, dependiendo de sus magnitudes; no obstante, las correlaciones genéticas entre peso o talla a distintas edades son bajas, aproximadamente, entre 0,3 y 0,5, para diferentes especies. Estudios de correlación genética entre peso corporal a diferentes edades en tilapia nilótica, han demostrado magnitudes mayores, que van de 0,65 a 1 (Turra et al. 2012) y que van en concordancia con los resultados obtenidos en el presente estudio.

En general, las correlaciones, tanto genéticas como fenotípicas entre peso y talla y entre variables a edades diferentes, fueron incrementándose, conforme se acrecentaba la edad; valores que sugieren que seleccionar a tempranos estados de desarrollo no es apropiado. Se podría obtener mayores pesos a edades avanzadas, como una respuesta correlacionada a la selección para peso a los 55 días de edad, incluso, considerando la baja estimación de la heredabilidad al comienzo del periodo de crecimiento, aunque fue incremental en función de la edad. Esta apreciación fue acorde a lo discutido por Turra et al. (2012), mencionando que podrían ser obtenidas mayores respuestas a la selección, cuando se realizaba a los 60 días de edad.

Para el análisis de los componentes de covarianza, el efecto promedio de talla, como variable principal y la variable peso, como covariable, mostró una mejor relación y respuesta en el análisis; un similar comportamiento se observó en estudio reportado por Eknath et al. (2007), en cuanto a las magnitudes: la correlación fenotípica fue siempre superior a la correlación genética. Las estimaciones del presente estudio pueden ser útiles en la construcción de índices de selección para la especie, si así se requiere, índices que pueden ser utilizados en programas de mejoramiento genético de especies acuícolas.

Para los efectos ambientales temporales o permanentes de los parentales que, generalmente están incluidos en la estimación de parámetros genéticos, se consideró disminuir dichos efectos del error en el modelo, para lo cual, se reprodujeron animales que tenían edades similares de reproducción, igual cantidad de desoves y sin parentesco alguno. En las progenies, no se consideró diferencia atribuible al sexo, por no existir dimorfismo en la especie. Además, los desoves fueron acordes con otros reportados, asegurando estimaciones en condiciones normales de producción.

En cuanto a la exactitud, es decir, menor desviación estándar de los parámetros genéticos depende de la cantidad de familias y no de la cantidad de progenies por familia y que la magnitud de la heredabilidad, usando datos de hermanos completos, como en este estudio, generalmente, es más alta que aquellos usando datos de hermanos medios; se debe tener cuidado en la interpretación de los datos, dando espacio a otros estudios similares, con los que se pueda contrastar. 
Como se ha mencionado, en especies como tilapia nilótica, (Oreochromis niloticus), trucha arcoíris (Oncorhynchus mykiss), salmón atlántico (Salmo salar), salmón coho (Oncorhynchus kisutch), carpa común (Cyprinus carpio), catfish (Ictalurus punctatus), rohu (Labeo rohita) y dorada (Sparus auratus L.), han sido implementados programas de mejoramiento con éxito, según lo describe Gjedrem et al. (2012), logrando importantes ganancias genéticas, para características de crecimiento, de producto final y de calidad; es por ello, que para establecer programas similares en yamú es necesario conocer la variación genética de características de interés comercial.

El estudio demostró, aun con limitaciones por el bajo número de familias obtenidas, que es posible estimar parámetros genéticos, para características de crecimiento en yamú.

Los parámetros genéticos estimados para peso y talla incrementaron, progresivamente, con la edad durante el periodo de estudio, quizás como resultado de una reducción gradual en la varianza de los efectos familiares. La correlación genética entre peso o talla, a diferentes edades del mismo individuo, sugiere una posible respuesta de selección correlacionada, a través del aumento en el periodo de crecimiento. Los resultados del estudio indican esperanzas para el fortalecimiento del conocimiento genético, no solo del yamú, sino también de otras especies del género Brycon, que puedan merecer un estudio similar. Además, se destaca el potencial de la industria del yamú, para mejorar estas características de crecimiento y de producción, no solo a través del manejo, sino de la explotación de la variación genética, una vez se avance en las áreas de la nutrición, la reproducción y la sanidad de la especie. Los resultados del estudio se deben interpretar cuidadosamente, puesto que no se sabe si la expresión de las características de crecimiento evaluadas, fuera del rango de edad del estudio, están influenciadas, en parte, por un grupo diferente de genes.

Conflicto de interés: El manuscrito fue preparado y revisado con la participación de todos los autores, quienes declaramos que no existe conflicto de interés que ponga en riesgo la validez de los resultados presentados.

\section{BIBLIOGRAFÍA}

1. ARIAS, J.A. 2006. Estado actual del conocimiento sobre el yamú, Brycon amazonicus. Rev. Col. Ciencias Pec. 19(2):125-133.

2. BECKER, W.A. 1975. Manual of Quantitative Genetics. Ed. Washington State University (Pullman, Washington ). 240p.
3. CABALLERO, J.G.; PABLO, E.J.; MARTÍNEZ, C.C. 2003. Estimación por máxima verosimilitud restringida de componentes de varianza y covarianza de múltiples características bajo los diseños I y II de Carolina del Norte. Fitotecnia Mexicana. 26(1):53-66.

4. CAMPOS, J.C. 2004. Melhoramento genético aplicado à produção animal. Ed. FEPMVZ (Belo Horizonte). $607 p$.

5. CARDELLINO, R.; ROVIRA, J. 1987. Mejoramiento Genetico Animal Montevideo. Ed. Agropecuária Hemisfério Sur (Uruguay). 253p.

6. CHARO-KARISA, H.; BOVENHUIS, H.; REZK, M.A.; PONZONI, R.W.; VAN ARENDONK, J.A.M.; KOMEN, H. 2007. Phenotypic and genetic parameters for body measurements, reproductive traits and gut length of Nile tilapia (Oreochromis niloticus) selected for growth in low-input earthen ponds. Aquaculture. 273(1):15-23.

7. DAZA, P.V.; LANDINES, M.A.; SANABRIA, A.I. 2005. Reproducción de peces en el trópico. Ed. Instituto Colombiano de Desarrollo Rural (INCODER) (Bogotá, Colombia). 246p.

8. DE SOUZA, E.C.M.; DA SILVA, J.P.; VILLACORTA-CORREA, M.A.; CARVALHO, T.B. 2014. Aggressiveness and locomotion activity related to hatching time in Matrinxã, Brycon amazonicus (Spix and Agassiz, 1829). Appl. Animal Behaviour Sci. 157:146-151.

9. EKNATH, A.E.; BENTSEN, H.B.; PONZONI, R.W.; RYE, M.; NGUYEN, N.H.; THODESEN, J.; GJERDE, B. 2007. Genetic improvement of farmed tilapias: Composition and genetic parameters of a synthetic base population of Oreochromis niloticus for selective breeding. Aquaculture. 273(1):1-14.

10. GALLEGO, F. 2001. Mejoramiento Genético en Acuicultura. En: Rodríguez, H., Daza, P.; Carrillo, M. (eds). Fundamentos de la Acuicultura Continental. Ed. Ministerio de Agricultura y Desarrollo Rural-Instituto Nacional de Pesca y Acuicultura-INPA (Bogotá, Colombia ). p.245-252.

11. GARCÍA-CELDRÁN, M.; RAMIS, G.; MANCHADO, M.; ESTÉVEZ, A.; NAVARRO, A.; ARMERO, E. 2015. Estimates of heritabilities and genetic correlations of raw flesh quality traits in a reared gilthead sea bream (Sparus aurata L.) population sourced from broodstocks along the Spanish coasts. Aquaculture. 446:181-186. 
12. GJEDREM, T. 2012. Genetic improvement for the development of efficient global aquaculture: A personal opinion review. Aquaculture. 344-349(0):12-22.

13. GJEDREM, T.; ROBINSON, N.; RYE, M. 2012. The importance of selective breeding in aquaculture to meet future demands for animal protein: A review. Aquaculture. 350-353(0):117-129.

14. HAFFRAY, P.; BUGEON, J.; RIVARD, Q.; QUITTET, B.; PUYO, S.; ALLAMELOU, J.M.; VANDEPUTTE, M.; DUPONT-NIVET, M. 2014. Reprint of: Genetic parameters of in-vivo prediction of carcass, head and fillet yields by internal ultrasound and 2D external imagery in large rainbow trout (Oncorhynchus mykiss). Aquaculture. 420-421, Supplement 1:S134-S142.

15. MALUWA, A.O.; GJERDE, B. 2006. Estimates of the strain additive, maternal and heterosis genetic effects for harvest body weight of an F2 generation of Oreochromis shiranus. Aquaculture. 259(1-4):38-46.

16. MCDONALD, J.H. 2008. Hand Book of Biological Statistics. Ed. Sparky (Baltimore, Maryland). 287p.

17. MERINO, M.; BONILLA, S.; BAGES, F. - AUINAP-FAOMinisterio de Agricultura y Desarrollo Rural. 2013. Diagnóstico del estado de la acuicultura en Colombia. Bogotá, Colombia. 160p. ISBN:978-958-579740-6.

18. NAVARRO, A.; ZAMORANO, M.J.; HILDEBRANDT, S.; GINÉS, R.; AGUILERA, C.; AFONSO, J.M. 2009a. Estimates of heritabilities and genetic correlations for body composition traits and $G \& \# x a 0 ; \times \& \# x a 0 ; E$ interactions, in gilthead seabream (Sparus auratus L.). Aquaculture. 295(3-4):183-187.

19. NAVARRO, A.; ZAMORANO, M.J.; HILDEBRANDT, S.; GINÉS, R.; AGUILERA, C.; AFONSO, J.M. 2009b. Estimates of heritabilities and genetic correlations for growth and carcass traits in gilthead seabream (Sparus auratus L.), under industrial conditions. Aquaculture. 289(3-4):225-230.

20. NGUYEN, V.; KLEMETSDAL, G.; ØDEGÅRD, J.; GJØEN, H.M. 2012. Genetic parameters of economically important traits recorded at a given age in striped catfish (Pangasianodon hypophthalmus). Aquaculture. 344-349(0):82-89.

21. SIPAÚBA-TAVARES, L.; ALVAREZ, E.; BRAGA, F. 2008. Water quality and zooplankton in tanks with larvae of
Brycon orbignyanus (Valenciennes, 1949). Braz. J. Biol. 68(1):77-86.

22. TURRA, E.M.; DE OLIVEIRA, D.A.A.; VALENTE, B.D.; TEIXEIRA, E.D.A.; PRADO, S.D.A.; DE MELO, D.C.; FERNANDES, A.F.A.; DE ALVARENGA, É.R.; E SILVA, M.D.A. 2012. Estimation of genetic parameters for body weights of Nile tilapia Oreochromis niloticus using random regression models. Aquaculture. 354-355(0):31-37.

23. URBINATI, E.C.; SARMIENTO, S.J.; TAKAHASHI, L.S. 2014. Short-term cycles of feed deprivation and refeeding promote full compensatory growth in the Amazon fish matrinxã (Brycon amazonicus). Aquaculture. 433:430-433.

24. VANDEPUTTE, M.; KOCOUR, M.; MAUGER, S.; RODINA, M.; LAUNAY, A.; GELA, D.; DUPONT-NIVET, M.; HULAK, M.; LINHART, O. 2008. Genetic variation for growth at one and two summers of age in the common carp (Cyprinus carpio L.): Heritability estimates and response to selection. Aquaculture. 277(1-2):713.

25. VÁSQUEZ, W. 2004. Principios de nutrición aplicada al cultivo de peces. Ed. Juan XXIII Ltda. (Universidad de los Llanos). 64p.

26. VELASCO-SANTAMARÍA, Y.; PALACIOS-RUIZ, C.; CRUZ-CASALLAS, P. 2008. Eficiencia anestésica de 2-fenoxietanol, benzocaina, quinaldina y metasulfonato de tricaina en alevinos y juveniles de cachama blanca (Piaractus brachypomus). MVZ Córdoba. 13(3):1435-1445

27. VINE, N.G.; LEUKES, W.D.; KAISER, H. 2004. In vitro growth characteristics of five candidate aquaculture probiotics and two fish pathogens grown in fish intestinal mucus. FEMS Microbiol. Letters. 231(1):145152.

28. WHATMORE, P.; NGUYEN, N.H.; MILLER, A.; LAMONT, R.; POWELL, D.; D'ANTIGNANA, T.; BUBNER, E.; ELIZUR, A.; KNIBB, W. 2013. Genetic parameters for economically important traits in yellowtail kingfish Seriola lalandi. Aquaculture. 400-401:77-84.

29. ZANIBONI-FILHO, E.; REYNALTE-TATAJE, D.; WEINGARTNER, M. 2006. Potencialidad del género Brycon en la piscicultura brasileña. Rev. Col. Ciencias Pec. 19(2):233-240. 
30. ZANUZZO, F.S.; ZAIDEN, S.F.; SENHORINI, J.A.; MAR- Recibido: Agosto 12 de 2015 ZOCCHI-MACHADO, C.M.; URBINATI, E.C. 2015. Aceptado: Enero 28 de 2016 Aloe vera bathing improved physical and humoral protection in breeding stock after induced spawning in matrinxã (Brycon amazonicus). Fish \& Shellfish Immunology. 45(1):132-140.

Cómo citar:

Bernal, F.; Gallego, F. 2016. Estimación de parámetros genéticos para peso y talla a diferentes edades en yamú (Brycon amazonicus). Rev. U.D.C.A Act. \& Div. Cient. 19(1): 123-130. 